\section{Real-time in vivo histologic examination using a probe-based endocytoscopy system for differ- entiating duodenal polyps}

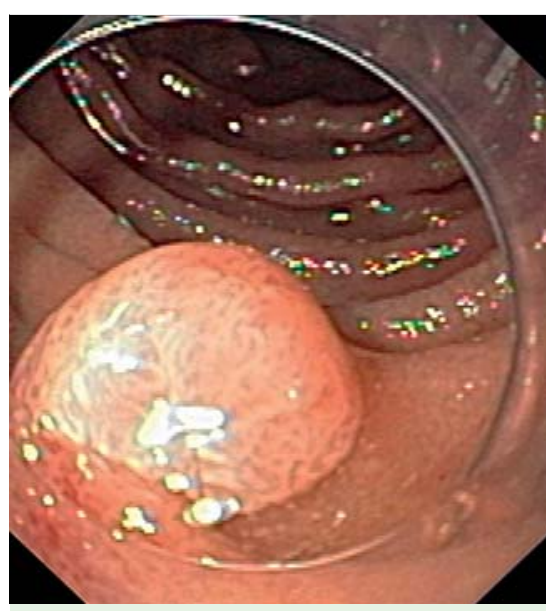

Fig. 1 Conventional white-light endoscopy in a 57-year-old-man with mild dyspeptic symptoms and occasional heartburn. There is a large polypoid lesion in the deeper part of the duodenum.

Endocytoscopy is an emerging endoscopic imaging modality enabling real-time in vivo visualization of the superficial mucosal layer at a magnification up to 1400 fold. This allows analyzing ultrastructural features of a tissue, including the arrangement and density of cells, the nucleus-tocytoplasm ratio, and the microvascular network [1]. Endocytoscopy is based on the principle of contact light microscopy and requires prestaining of the mucosa with either methylene blue or toluidine blue. Previous studies have shown the potential of endocytoscopy for in vivo diagnosis of esophageal squamous cell carcinoma, celiac disease, and colonic polyps. Moreover, recent data suggest the potential of endocytoscopy to differentiate between individual mucosal inflammatory cells during endoscopy [2-4].

Here, we present the case of a 57-year-oldman, who presented at our outpatient clinic with mild dyspeptic symptoms and occasional heartburn. Physical examination, medical history, and routine laboratory investigations were unremarkable. Esophagogastroduodenoscopy (EGD) showed an 8-mm polypoid lesion in the deeper part of the duodenum ( $\bullet$ Fig. 1 ). After withdrawal of the endoscope (GIF Q160; Olympus, Tokyo, Japan) we attached a clear cap to the distal end of the scope. After thorough washing of the mucosa with water, targeted chromoendoscopy was used to apply $1 \%$ methylene blue over the polypoid lesion. To allow good differentiation of mucosal cells, the stain was left in situ for 3 minutes, followed by repeat washing of the mucosa with water. Then a probebased endocytoscopy system (XEC-300,
Olympus, Tokyo, Japan) was introduced through the working channel of the endoscope until the top of the endocytoscope touched the polyp's surface to ensure in vivo tissue imaging. The polypoid duodenal mucosa showed elongation of glands and villi and a slightly serrated appearance. In addition, we observed several small, roundish nuclei, but these were not indicative of malignancy ( Fig.2). The polyp was resected by snare polypectomy and the in vivo diagnosis of hyperplastic regenerative polyp was confirmed on histopathological analysis, which showed hyperplastic epithelium, elongated glands, incomplete gastric metaplasia of the surface epithelium, increase in the number of capillaries, and apical fibrosis (mostly due to healing of a prior lesion). A few hyperplastic Brunner's glands were present in the lower half of the lesion and extending towards the surface, thereby further enhancing the polypoid aspect of the lesion (๑ Fig. 3).

Our case is of interest for several reasons. First, this is the first time an in vivo optical biopsy technique has been used for characterization of a duodenal polyp. Endocytoscopy readily identified mucosal alterations typical of a hyperplastic regenerative duodenal polyp. Second, several types of polyps can occur in the duodenum, including non-neoplastic (e.g., ectopic gastric mucosa, inflammatory polyps, Brunner's gland hyperplasia) and neoplastic (e.g., adenoma, gastrointestinal stromal tumor) polyps. The reported frequency of occurrence of hyperplastic duodenal polyps is very low. To the best of our

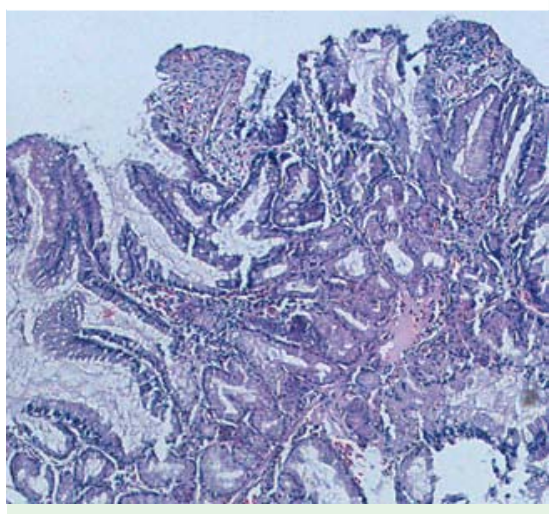

Fig.3 Histopathologic section showing hyperplastic epithelium, elongated glands, incomplete gastric metaplasia of the surface epithelium, increase in the number of capillaries, and apical fibrosis due principally to healing of a previous lesion. The hyperplastic Brunner's glands in the lower half of the lesion are extending towards the surface, further enhancing the polypoid aspect of the lesion. 
knowledge only three cases have been reported [5]. Hyperplastic duodenal polyps mostly occur in the setting of ectopic gastric mucosa and may be associated with colonization of Helicobacter pylori. In addition, we provide a detailed description of the technical procedure, which may assist further use of the technique. Third, our report adds to the growing literature on the use of advanced endoscopic methods for small-bowel imaging. Lastly, we have shown that in vivo imaging using endocytoscopy is relatively easy to carry out during an endoscopic examination, with immediate access to "histological" findings, which may allow clinical decisions to made with minimal time delay.

Endoscopy_UCTN_Code_CCL_1AB_2AZ_3AB

Competing interests: None

\section{H. Neumann' ${ }^{1}$ S. Kudo ${ }^{2}$, M. Vieth ${ }^{3}$, M. F. Neurath ${ }^{1}$}

${ }^{1}$ Department of Medicine I, University of Erlangen-Nuremberg, Erlangen, Germany 2 Digestive Disease Center, Showa University Northern Yokohama Hospital, Japan

${ }^{3}$ Institute of Pathology, Klinikum Bayreuth, Bayreuth, Germany

\section{References}

1 Neumann H, Fuchs FS, Vieth $M$ et al. Review article: in vivo imaging by endocytoscopy. Aliment Pharmacol Ther 2011; 33: 1183 1193

2 Kudo SE, Wakamura K, Ikehara $N$ et al. Diagnosis of colorectal lesions with a novel endocytoscopic classification - a pilot study. Endoscopy 2011; 43: 869-875

3 Pohl H, Rösch T, Tanczos BT et al. Endocytoscopy for the detection of microstructural features in adult patients with celiac sprue: a prospective, blinded endocytoscopy-conventional histology correlation study. Gastrointest Endosc 2009; 70: 933 -941
4 Neumann $H$, Vieth $M$, Neurath $M F$ et al. Endocytoscopy allows accurate in vivo differentiation of mucosal inflammatory cells in IBD: A pilot study. Inflamm Bowel Dis 2012, [Epub ahead of print]

5 Roche HJ, Carr NJ, Laing H et al. Hyperplastic polyps of the duodenum: an unusual histological finding. J Clin Pathol 2006; 59: 1305-1306

\section{Bibliography}

DOI http://dx.doi.org/

10.1055/s-0032-1325970

Endoscopy 2013; 45: E53-E54

(c) Georg Thieme Verlag KG

Stuttgart · New York

ISSN 0013-726X

\section{Corresponding author}

\section{H. Neumann}

Department of Medicine I

University of Erlangen-Nuremberg

Ulmenweg 18

91054 Erlangen

Germany

Fax: +49-9131-8535209

helmut.neumann@uk-erlangen.de 